\title{
La vie de la Société
}

Lors du XIIème Congrès de la SALF qui s'est tenu à Tours en Décembre 1995, trois posters ont reçu un prix de 5000F (Laboratoires Pharmagyne, Synthelabo, et SALF) : il s'agit des travaux présentés respectivement par J.Auer, H.Lejeune et J.M.Rigot, dont les textes figurent dans le présent numéro d'Andrologie.

L’Assemblée Générale annuelle de notre Société, tenue lors de ce XIIème Congrès, a donné lieu à l'arrivée de trois nouveaux membres au sein du CA, Jacques AUGER, Serge CARREAU et Jean-Marc RIGOT,deux autres membres ayant leur mandat renouvelé : Henri NAVRATIL et Charles SULTAN. La nouvelle composition du Conseil d'Administration figure en page 2 de ce numéro.

L'année 1996 verra la SALF participer activement à ou organiser différentes manifestations.

Du 12 au 14 Avril 1996, la SALF participera aux 3ème Journées de la Société Marocaine d'Andrologie et de Sexologie (SMAS) à Casablanca, organisées par la SMAS avec la collaboration scientifique de l'Association Marocaine de Sexologie (AMS, l'Association Inter-hospitalo-Universitaire de Sexologie (AIHUS) et les Cecos Alsace et Midi-Pyrénées.

Du 8 au 10 Mai 1996 aura lieu au Palais des Congrès à Paris le premier Congrès de la Fédération Française d'Etude de la Reproduction (FFER), fédération qui regroupe 9 Sociétés impliquées dans la reproduction, dont la SALF. Notre Société, en collaboration avec l'Association Française d'Urologie (AEU) et la Fédération des CECOS, traitera plus particulièrement deux thèmes lors de l'aprèsmidi du jeudi 9 mai : Cryptorchidie et Infertilité ainsi que Cancer du testicule et Infertilité.

Le XIIIème Congrès de la SALF se tiendra du 12 au 14 décembre 1996 à Nancy. Après une série d'ateliers (maladie de Lapeyronie, andropause, biochimie du sperme, température testiculaire), les principaux thèmes abordés seront la pathologie de la puberté, la sexualité du mâle (nouvelles approches), les nouveaux débats sur la spermatogénèse, procréation et paraplégie, ainsi que le traitement des troubles de l'érection. Parallèlement, le Club Français du Testicule tiendra sa rencontre annuelle le jeudi 12 décembre 1996. L’assemblée générale annuelle de notre Société aura lieu le vendredi 13 décembre 1996.

Enfin, le XIVème Congrès de la SALF, qui aura lieu en décembre 1997, est déjà en préparation, et un pré-programme paraîtra dans le numéro de juin 1996 d'Andrologie. 


\section{PROGRAMME PROVISOIRE DU}

\section{XIIIème CONGRES DE LA SALF \\ NANCY 12-13-14 Décembre 1996}

Palais des Congrès Rue du Grand Rabbin Haguenauer 54000 NANCY Contact : Hubert GERARD, Laboratoire d'Histologie-Embryologie Faculté de Médecine (83 5928 65, Fax : 835928 83) et Maternité A.PINARD

\section{Journée du 12 Décembre 1996}

Matinée 12 Décembre

\section{ACTUALITES - MISES AU POINT - ATELIERS (3-4h)}

Maladie de la Peyronie

(T.Amar, M.Schouman)

Andropause

(H.Lejeune, J.Belaisch)
Biochimie du sperme

(S.Hamamah, J.C.Soufir)

Hyperthermie testiculaire

(P.Jouannet, R.Mieusset)

Après-midi 12 Décembre

\section{ADOLESCENCE - PATHOLOGIE DE LA PUBERTE}

(2h)

La puberté surrénalienne (M.Forest)

Le sperme à l'adolescence (P.Jouannet)

Psychologie de l'adolescent (D.Marcelli)

Le micro pénis à la puberté (C.Sultan)

SEXUALITE DU MALE : NOUVELLES APPROCHES (2h)

Modélisation du comportement sexuel chez le rat (O.Rampin)

Neurophysiologie centrale de la fonction sexuelle chez le rat mâle

(K.Mc Kenna USA)

Cerveau et sexualité masculine (S.Stoleru) 


\section{Journée du 13 Décembre 1996 \\ SPERMATOGENESE : LES NOUVEAUX DEBATS}

Matinée

Les cellules spermatogénétiques sont-elles sous contrôle direct des stéroïdes sexuels ? (3h)

Présence de récepteurs aux androgènes sur la lignée germinale : état de la question

(Suarez-Quiang, USA)

Activité aromatase dans les cellules germinales mâles et présence de récepteurs à l'oestradiol : quelle signification fonctionnelle? (S.Carreau)

Manipulation des gènes des protéines de transports des stéroïdes sexuels et anomalie de la spermatogénèse

(Reventos, Espagne)

Déambulation posters (1h30)

Après-midi :

Prolifération et méiose : conditions nécesaires et suffisantes pour la fertilité masculine $(3 \mathrm{~h})$

Fécondance du gamète mâle immature : réalité scientifique, perspectives thérapeutiques, problèmes éthiques.

Expression de facteurs de croissance et de leurs récepteurs par les cellules spermatogéniques : rôles dans le contrôle de la prolifération de la lignée germinale (Benhamed)

Les mécanismes de contrôle de la méiose dans la lignée germinale mâle : état de la question (Durand)

Testicule et infection : quelles conséquences pour la spermatogénèse (N....)

La réponse du testicule à l'infection : nature des cytokines mises en jeu, mécanisme de leur régulation et implications cliniques de leur impact sur la spermatogénèse (B.Jégou)

\section{ASSEMBLEE GENERALE SALF}

SOIREE DE GALA 


\section{Matinée 14 Décembre}

Procréation et paraplégie Table ronde (1h30)

Anatomie physiologie (J.Hubert)

Physiopathologie (Brugerolle)

Prise en charge globale (P.Costa)

Résultat des techniques de PMA (F.Guillet-May)

Séance meilleurs posters (1h)

Traitement des troubles de l'érection (1h)

Injections intracaverneuses. Résultats à long terme (J.Buvat)

Les autres traitements pharmacologiques (F.Giuliano)

\section{JOURNEE SATELLITE}

\section{Décembre}

Réunion du Club Français du Testicule

\section{Contact :}

Anne GERARD Laboratoire d'Histologie-Embryologie

Faculté de Médecine

Tél : 89.59.28.93,

Fax : 83.59.28.83 